\title{
Optimal Relay Selection and Beamforming Strategy in Cooperative Systems with Limited Feedback and Asymptotically Large Number of Relays
}

\author{
Yuxian Zhang, Roger S. Cheng and Yin Yang \\ Department of Electronic and Computer Engineering \\ The Hong Kong University of Science and Technology \\ Email: \{eegabby, eecheng, yangyin\}@ust.hk
}

\begin{abstract}
We consider a cooperative wireless network with a single source, a single destination, and $M$ cooperative relays. We show that, with only limited feedback, $M \bar{B}$ bits, from the destination, using all the relays to perform beamforming may not be optimal. Assuming that the destination selects the relays whose power gains exceed a threshold and feeds back $M \bar{B}$ bits to specify a precoding matrix from a randomly generated precoder codebook, we find the optimal threshold that maximizes the capacity of a Decode-and-Forward (DF) system for a given $\bar{B}$, asymptotically in the number of relays, $M$. We show that the system using the optimal threshold has a higher capacity than a system using all relays for all $\bar{B}$, especially for small values of $\bar{B}$. Furthermore, using a fixed fraction $\left(\beta^{*}\right.$ which is obtained from the optimal threshold) of relays in systems with finite number of relays also leads to a significant improvement in outage probability, comparing to that of the systems using all relays, in both DF and Amplify-and-Forward (AF) cooperating schemes.
\end{abstract}

Index Terms-Relay selection, beamforming, large system, limited feedback, relay ordering

\section{INTRODUCTION}

$\mathbf{I}$ $\mathrm{N}$ wireless cooperative networks, diversity order can be increased even without having multiple antennas at each node, which makes it more practical for small mobile equipments. Various cooperative approaches have been studied, including space-time coding, power allocation, relay selection, and beamforming (e.g. [1]-[4]), under different amount of channel knowledge and conditions.

In the amplify-and-forward (AF) relay networks, singlerelay selection schemes have been studied extensively (e.g., [2],[4]), and those based on SNR, harmonic mean, and the worst link quality, have been proved to achieve full diversity order [3]. However, quite a large gap in coding gain exists between the performance of using single-relay selection and the optimal beamforming which involves all relays with unlimited feedback.

While the optimal performance in beamforming comes at the cost of unlimited feedback which seems to be impractical for the sake of power consumption and delay, the large gap motivates the study of the performance when feedback bits are limited. The second phase of decode-and-forward (DF) networks can be regarded as a conventional multiple-input single-output (MISO) transmission problem. [6] provides a good perspective and results by generalizing the problem from the large system's point of view. By employing Random Vector Quantization (RVQ) as the precoding matrix with $\bar{B}$ feedback bits per available transmit antenna, [6] has derived the asymptotic capacity in term of $\bar{B}$ explicitly. Nevertheless, when $\bar{B}$ becomes small, the performance decays rapidly. On the other hand, numerical examples illustrate that HybridSelection/Maximal Ratio Combining, even with $L \ll N$, can enjoy performance close to that of $N$-branch MRC [8]. Hence it is of interest to find the optimal fraction of antennas/relays that should be used under limited feedback resource.

The authors in [10] suggested to allow more than one relay to participate and identified two multiple relay-selection schemes in the AF mode. However, they required partial transmitter-side channel state information (CSI) at relays to adjust the phase. But the cost of attaining the transmitter-side CSI has been found to be significant [9].

In this paper, we make the practical assumption that no CSI is available at the transmitter. The amount of feedback containing precoding information is fixed to be $B$ bits. We first work on the model of DF networks and empoloy the idea of selecting relays with their power gains exceeding a threshold, which guarantees a fixed fraction $\beta$ almost surely asymptotically, to be active. The RVQ, which has been proved to be "asymptotically optimal" [6], is employed for the precoding matrix design. The asymptotic capacity is derived as a function of the fraction $\beta$ for a given $\bar{B}$, the number of feedback bits per selected relay. Then a scheme which always selects a fixed fraction of relays is inspired by the asymptotic result. Subsequently, a similar fixed fraction scheme is adopted to AF networks. A relay ordering method is introduced, motivated by the works in [2]. Simulation results show the optimal $\beta$ derived for asymptotic results can be used in the finite cases to achieve close to optimal outage performance which provides a significant improvement compared to using all relays in both $\mathrm{DF}$ and AF networks.

The rest of the paper is organized as follows. The system model is introduced in Section II. In Section III, the "Threshold Selection (TS)" is proposed in the DF network, and the asymptotic analysis is presented. Section IV introduces the "Fraction Selection (FS)"scheme in the finite case which is motivated by the asymptotic result, and gives the simulation results. Then FS is applied in the AF network in Section V, and the performance is examined as well. Conclusion is made 
in Section VI.

\section{SYSTEM MODEL}

Consider a cooperative network where a source transmits to a destination with the help of $K$ relay nodes of $\mathrm{DF}$ Mode. Every node has a single antenna. All channel gains are independent and identically distributed (i.i.d.) Gaussian random variables with unit variance. Here the direct link is not taken into consideration. At all nodes, the additive white Gaussian noise contributions are also i.i.d. with single-sided power spectral density $N_{0}$. In this scenario, we assume global CSI at the destination while the relays have no knowledge about the channels from relays to the destination. Instead, totally $B$ bits are available at the destination to feed back some precoding information to the relays.

In Phase I, the source node broadcasts the unit power signal $x$ with energy $E_{s}$. Relay $k$ receives the signal

$$
r_{k}=\sqrt{E_{s}} g_{k} x+n_{k},
$$

where $g_{k}$ is the channel coefficient from the source to the $k$ th relay, and $n_{k}$ is the corresponding additive white Gaussian noise. We assume that totally $M$ relays can fully decode the message. Now we will focus only on these $M$ relays and their corresponding channel responses to the destination, denoted by $h_{m}, 1 \leq m \leq M$. Using the global CSIR, the destination selects $N(1 \leq N \leq M)$ relays and invites the selected relays to participate in Phase II. All other relays will be in sleep mode in Phase II. The transmission energy in Phase II is fixed to be $E_{r}$, which will be shared among the selected relays. A RVQ codebook with $2^{B}$ candidates $\mathcal{W}=\left\{\mathbf{w}_{1}, \ldots, \mathbf{w}_{2^{B}}\right\}$ is known a priori to both the destination and the relays, where $\mathbf{w}_{j}$ is a $N \times 1$ vector with $\left\|\mathbf{w}_{j}\right\|^{2}=1$ to satisfy the energy constraint, for $1 \leq j \leq 2^{B}$. The destination then chooses the $\mathbf{w}_{j}$ that will maximize the received SNR, i.e.,

$$
\gamma_{r}=\left|\mathbf{h}^{\dagger} \mathbf{w}_{j}\right|^{2} \gamma_{t}
$$

where $\gamma_{t}=E_{r} / N_{0}, \mathbf{h}$ is a $N \times 1$ vector with the $n$th entry the channel coefficient between the $n$th selected relay and the destination. Let the selected precoding matrix be

$\mathbf{w}^{*}=\arg \max _{1 \leq j \leq 2^{B}}\left\{\gamma_{r}=\gamma_{t}\left|\mathbf{h}^{\dagger} \mathbf{w}_{j}\right|^{2}\right\}=\arg \max _{1 \leq j \leq 2^{B}}\left\{\left|\mathbf{h}^{\dagger} \mathbf{w}_{j}\right|^{2}\right\}$.

The index of $\mathbf{w}^{*}$ is fed back to all the selected relays through an error-free side-channel in $B$ bits. In Phase II, all the selected relays transmit simultaneously to the destination, with the $n$th relay weighting $x$ with the $n$th entry of $\mathbf{w}^{*}$. The received beamforming signal at the destination becomes

$$
r_{d}=\sqrt{E_{r}} \mathbf{h}^{\dagger} \mathbf{w}^{*} x+n_{d},
$$

where $n_{d}$ is the white Gaussian noise at the destination in the second phase. The maximal received SNR is defined as

$$
\gamma_{r}^{N} \triangleq \max _{1 \leq j \leq 2^{B}} \gamma_{t}\left|\mathbf{h}^{\dagger} \mathbf{w}_{j}\right|^{2}
$$

Note that choosing none of the relays $(N=0)$ is apparently non-optimal, and $N=M$ is equivalent to choosing all relays. It is important to note that all selected relays share the same channel (exactly the same time and frequency band) to perform beamforming in this model. This is different from a lot of existing papers which assumed that different relays use different channels and in those papers, space time codes are often used instead of beamforming.

As global CSI is available at the destination, exhaustive search can be used to find the best subset of relays for beamforming. However, the complexity of exhaustive search grows exponentially in $M$. It will be beneficial if we can find a low complexity selection scheme with close to optimal performance. In this work, we analyze the asymptotic problem and use the optimal asymptotic solution to derive a simple selection scheme for systems with finite number of relays.

Note that although a direct link is not considered here, it plays no role in the selection scheme. Even if the channel from the source to the destination exists, it will have no impact on the comparison among different selections, i.e. how many and which relays should be chosen to achieve best performance remain the same.

\section{THRESHOLD SELECTION OF THE DECODE-AND-FORWARD NETWORK IN THE ASYMPTOTICALLY LARGE SYSTEM}

For the benefit of effective transmission, we tend to use relays with the largest channel gains. Let $\mathbf{f}$ be an $M \times 1$ vector with the entries the channel coefficients $h_{m}$ from all the relays to the destination. Then $\mathbf{h}$ is the $N \times 1$ sub-vector formed by the $N$ entries of $\mathbf{f}$ with the largest $\left|h_{m}\right|^{2}$.

For fixed $M, \gamma_{r}^{N}$ depends on $N$ and the channel responses and the codebook. As in [6], we consider the problem with asymptotically large number of relays. Considering the asymptotic case not only allows us to deal with the feedback more conveniently, but also makes solving the Hybrid Selection problem much more easily. We let the number of available relays $M$ and the number of feedback bits $B$ go to infinity with fixed $\bar{B}=B / M$ (feedback bits per available relay). If the channel coefficients of the selected relays are not i.i.d., it is hard to analyze the performance under RVQ. In the large system, we use the "Threshold Selection (TS)" method with a predetermined power gain threshold $r$ and the destination only selects relays with $\left|h_{m}\right|^{2} \geq r$. This ensures that all channel coefficients of the selected relays are i.i.d., making the analysis manageable.

Lemma 1: Let $r$ satisfy $\int_{r}^{\infty} e^{-y} \mathrm{~d} y=\beta(r=-\ln \beta)$. As $M \rightarrow \infty$, we have that $N / M \stackrel{\text { a.s. }}{\longrightarrow} \beta$, i.e., the fraction of the relays selected under the TS scheme converges to $\beta$ almost surely.

The proof of this Lemma is a direct consequence of the property of Binomial distribution. In other words, in the large system, the TS scheme guarantees a fixed fraction of selected relays with probability $1 . \beta$ is the corresponding fraction which has a 1-to-1 correspondence to the threshold. In the following, we mostly use $\beta$ instead of $r$ in our analysis.

For simplicity, just consider the transmission in Phase II now. The optimal beamformer for systems is the normalized channel vector $\mathbf{f} /|| \mathbf{f} \|$, resulting in the all-participate optimal 
mutual information $I_{a p-o p t}=E_{\mathbf{f}}\left[\log _{2}\left(1+\gamma_{t} \mathbf{f}^{\dagger} \mathbf{f}\right)\right]$. As $M \rightarrow$ $\infty, \mathbf{f}^{\dagger} \mathbf{f} / M \stackrel{\text { a.s. }}{\longrightarrow} 1$, so $I_{a p-o p t}-\log _{2}\left(\gamma_{t} M\right) \stackrel{\text { a.s. }}{\longrightarrow} 0$ for any non-zero $\gamma_{t}[6]$. This can be fulfilled when there is perfect CSI at the relay-side, or equivalently $\bar{B}$ is infinity. Here we use the mutual information of all-participate perfect beamforming as our reference, and let the difference between the mutual information under TS with $\beta$ and $\log _{2}\left(\gamma_{t} M\right)$, i.e.,

$$
\Delta I^{\beta} \triangleq I^{\beta}-\log _{2}\left(\gamma_{t} M\right)
$$

be the comparison metric, where

$$
I^{\beta} \triangleq \max _{1 \leq j \leq 2^{B}}\left\{\log _{2}\left(1+\gamma_{t}\left|\mathbf{h}^{\dagger} \mathbf{w}_{j}\right|^{2}\right)\right\} .
$$

Lemma 2: Let $\mathbf{h}$ be the $N \times 1$ vector whose entries are the channel coefficients with power gains exceeding the threshold $r$, as $M \rightarrow \infty, \frac{\|\mathbf{h}\|^{2}}{M}$ converges to $\beta(1-\ln \beta)$ almost surely.

Proof: $h_{1}, h_{2}, \ldots, h_{M}$, are i.i.d. complex Gaussian random variables with unit variance. Thus, all $y_{m}=\left|h_{m}\right|^{2}(1 \leq$ $m \leq M)$ are exponential random variables with distribution $e^{-y_{m}}, 0 \leq y_{m} \leq \infty$. Let $\mathbf{z}$ be an $M \times 1$ vector with the entries $y_{m}$.

To get $\mathbf{h}$, first we can get

$$
\mathbf{z}^{\prime}=(\mathbf{z}-r)^{+},
$$

where

$$
x^{+}= \begin{cases}x & \text { if } x>0 \\ 0 & \text { if } x \leq 0 .\end{cases}
$$

Then remove all the $0 \mathrm{~s}$ from $\mathbf{z}^{\prime}$ to derive $\mathbf{y}$, the corresponding $h_{m}$ to the entries of $\mathbf{y}$ forms $\mathbf{h}$. In fact, all the disordered entries in $\mathbf{y}$ are i.i.d. random variables with distribution $\frac{e^{-y}}{\beta}, r<y<\infty$. Since $N / M \stackrel{\text { a.s. }}{\longrightarrow} \beta$,

$$
\begin{aligned}
\lim _{M \rightarrow \infty} \frac{\|\mathbf{h}\|^{2}}{M} & =\lim _{M \rightarrow \infty} \frac{N E[y]}{M} \\
& =\lim _{M \rightarrow \infty} \frac{N}{M} \int_{r}^{\infty} \frac{e^{-y}}{\beta} y \mathrm{~d} y \\
& =\beta(1-\ln \beta)
\end{aligned}
$$

almost surely.

Lemma 3: In the large system, given the fixed fraction of selected relays $(\beta)$, the Threshold Selection with RVQ for the precoding matrix is the asymptotically optimal selection scheme that maximizes the capacity.

Proof: Let $X_{j}=\frac{\left|\mathbf{h}^{\dagger} \mathbf{w}_{j}\right|^{2}}{\|\mathbf{h}\|^{2}}$. All the entries of $\mathbf{h}$ are still i.i.d. random variables, therefore in

$$
\left|\mathbf{h}^{\dagger} \mathbf{w}_{j}\right|^{2}=\|\mathbf{h}\|^{2} \frac{\left|\mathbf{h}^{\dagger} \mathbf{w}_{j}\right|^{2}}{\|\mathbf{h}\|^{2}}=\|\mathbf{h}\|^{2} X_{j},
$$

$\|\mathbf{h}\|^{2}$ and $X_{j}$ are independent [11]. Consequently, to maximize $I^{\beta}$ is equivalent to maximize $\|\mathbf{h}\|^{2}$ and $X_{j}$ respectively. It has been proven in [6] that the upper bound of $X_{j}$ can be achieved when applying RVQ for precoding matrix in the large system. On the other hand, when $\beta$ (the proportion of selected relays in the large system) is fixed, maximizing $\|\mathbf{h}\|^{2}$ is equivalent to choosing the channels with largest power gains. Thus, selecting the relays whose corresponding channel power gains to the destination exceed the threshold that satisfies the fixed proportion of selected relays $(\beta)$ is the asymptotically optimal selection scheme.

Following these results and that in [6], we can get the explicit asymptotic result of this Threshold Selection scheme.

Theorem 1: As $M, B \rightarrow \infty$ with fixed $\bar{B}=B / M$, with the threshold that results in the fixed subset proportion of selected relays $(\beta), \Delta I^{\beta}$ converges in the mean square sense to

$$
\Delta \mathbb{I}^{\beta}=\lim _{(M, B) \rightarrow \infty} \Delta I^{\beta}=\log _{2}\left[\beta(1-\ln \beta)\left(1-2^{-\frac{\bar{B}}{\beta}}\right)\right] .
$$

Proof: Given $\mathbf{h}$, since all $\mathbf{w}_{j}$ are i.i.d., $X_{j}$ are i.i.d., $j=1,2, \ldots, 2^{B}$. Then based on the analytical result from Appendix A of [6], similar result can be driven here expect that now only a proportion of relays are participating the transmission and that the number of feedback bits per used relay is $\hat{B}=\bar{B} / \beta=B / N$ almost surely. It gives

$$
\lim _{M \rightarrow \infty} E_{w}\left[\max _{j} X_{j} \mid \mathbf{h}\right]=1-2^{-\hat{B}}=1-2^{-\frac{\bar{B}}{\beta}} .
$$

Indeed, given $\mathbf{h}, \max _{j} X_{j} \rightarrow 1-2^{-\frac{\bar{B}}{\beta}}$ in the mean square sense [6]. As $\hat{B}$ increases, $\max _{j} X_{j}$ increases, and this demonstrates the positive effect by using less relays and thus making more feedback resource per selected relay when $\bar{B}$ is fixed.

Combined with Lemma 2, the asymptotic rate difference converges to

$$
\begin{aligned}
\Delta \mathbb{I}^{\beta} & =\lim _{(M, B) \rightarrow \infty}\left(\log _{2}\left(1+\gamma_{t}\|\mathbf{h}\|^{2} \max _{j} X_{j}\right)-\log _{2}\left(\gamma_{t} M\right)\right) \\
& =\lim _{(M, B) \rightarrow \infty} \log _{2}\left(\frac{1}{\gamma_{t} M}+\frac{\|\mathbf{h}\|^{2}}{M} \max _{j} X_{j}\right) \\
& \left.=\lim _{(M, B) \rightarrow \infty} \log _{2}\left(\frac{\|\mathbf{h}\|^{2}}{M} \max _{j} X_{j}\right)\right] \\
& =\log _{2}\left[\beta(1-\ln \beta)\left(1-2^{-\frac{\bar{B}}{\beta}}\right)\right]
\end{aligned}
$$

in the mean square sense.

Theorem 2: For each $\bar{B}$, there is only one optimal fraction, say $\beta^{*}$, which is characterized by $\ln \beta^{*}\left(\ln 2-\frac{\beta^{*}}{B}\left(2^{\frac{\bar{B}}{\beta^{*}}}-1\right)\right)-$ $\ln 2=0$, or equivalently a unique threshold $r^{*}=-\ln \beta^{*}$, to maximize the asymptotic capacity.

Proof: For a fixed $\bar{B}$, take derivative of $\Delta \mathbb{I}^{\beta}$ in terms of $\beta$,

$$
\begin{aligned}
\frac{\mathrm{d} \Delta \mathbb{I}^{\beta}}{\mathrm{d} \beta} & =\frac{\mathrm{d}}{\mathrm{d} \beta} \log _{2}\left[\beta(1-\ln \beta)\left(1-2^{-\frac{\bar{B}}{\beta}}\right)\right] \\
& =\frac{\ln \beta\left(\ln 2-\frac{\beta}{B}\left(2^{\frac{\bar{B}}{\beta}}-1\right)\right)-\ln 2}{(\ln 2) \beta(1-\ln \beta) \frac{\beta}{\bar{B}}\left(2^{\frac{\bar{B}}{\beta}}-1\right)} \\
& \stackrel{\alpha}{ } \stackrel{\triangleq \frac{\beta}{B}}{=} \frac{\ln \beta\left(\ln 2-\alpha\left(2^{\frac{1}{\alpha}}-1\right)\right)-\ln 2}{(\ln 2) \beta(1-\ln \beta) \alpha\left(2^{\frac{1}{\alpha}}-1\right)}
\end{aligned}
$$

where $\beta \in(0,1], \alpha \in\left(0, \frac{1}{B}\right)$, and it is easy to find that the denominator is always positive.

The numerator is always a monotonically decreasing function of $\beta$ when $\beta \in(0,1]$, ranged from $+\infty$ to $-\ln 2$. Reasons are shown below.

Let $f(\alpha)=\alpha\left(2^{\frac{1}{\alpha}}-1\right)$. When $\beta \rightarrow 0, \alpha \rightarrow 0, \ln \beta \rightarrow-\infty$, $f(\alpha) \rightarrow+\infty, \ln 2-f(\alpha) \rightarrow \infty$, so the numerator goes to $+\infty$ as a whole. When $\beta \rightarrow 1, \alpha \rightarrow 1 / \bar{B}$, then $\ln \beta \rightarrow 0$, 
$f(\alpha)=\frac{2^{\bar{B}}-1}{\bar{B}}$ is constant (in fact, when $\alpha \rightarrow \infty$ as $\bar{B} \rightarrow 0$, $\left.\lim _{\alpha \rightarrow \infty} f(\alpha)=\ln 2\right), \ln 2-f(\alpha)$ is constant $\left(\lim _{\alpha \rightarrow \infty} \ln 2-\right.$ $f(\alpha)=0)$, the numerator tends to $-\ln 2$.

$f(\alpha)^{\prime}=2^{\frac{1}{\alpha}}-(\ln 2) \cdot \frac{2^{\frac{1}{\alpha}}}{\alpha}-1$, we can derive $\lim _{\alpha \rightarrow 0} f(\alpha)^{\prime}=$ $-\infty, \lim _{\alpha \rightarrow \infty} f(\alpha)^{\prime}=0$ and $f(\alpha)^{\prime \prime}=\frac{2^{\frac{1}{\alpha}}(\ln 2)^{2}}{\alpha^{3}}>0$ for $\alpha \in$ $(0, \infty)$. So $f(\alpha)^{\prime}$ is always negative and $f(\alpha)$ is always a convex decreasing function of $\alpha$. Because $\alpha=\beta / \bar{B}$ and $\bar{B}>$ $0, \ln 2-f(\alpha)=\ln 2-f(\beta / \bar{B})$ is also an increasing function in terms of $\beta$. While $\ln \beta$ is non-positive increasing function of $\beta$ when $\beta \in(0,1]$, the numerator as a whole is always a decreasing function of $\beta$, ranged from $+\infty$ to $-\ln 2$.

In conclusion, $\Delta \mathbb{I}^{\beta}$ is increasing with $\beta$ in $\left(0, \beta^{*}\right)$ and decreasing in $\left(\beta^{*}, 1\right]$. $\beta^{*}$ is the only maximum point for $\Delta \mathbb{I}^{\beta}$. In fact, $\beta^{*}$ increases when $\bar{B}$ increases, and $\beta^{*} \rightarrow 1$ as $\bar{B} \rightarrow \infty$ which accords with the situation that if unlimited feedback is available, we should use all relays.

Consequently, based on $\bar{B}$, the TS with RVQ for precoding matrix and the corresponding $\beta^{*}$ is the asymptotically optimal selection scheme. Fig. 1 shows the $\beta^{*}$ for different $\bar{B}$ s, and Fig. 2 presents the asymptotic mutual information difference between TS with $\beta^{*}$ and the optimal beamforming, comparing with that of all-participate scheme $(\beta=1)$ [6]. The gain from applying Threshold Selection scheme increases as $\bar{B}$ decreases.

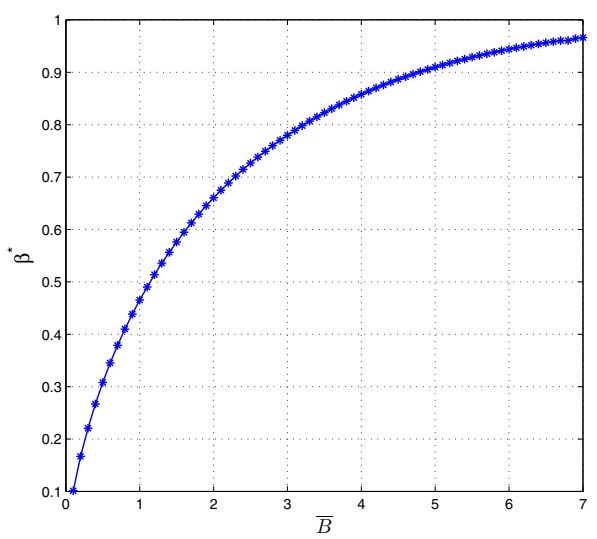

Fig. 1. Optimal $\beta$ vs. $\bar{B}$.

\section{ApPliCATION IN The Finite System AND SiMULATION RESULTS}

Providing $\bar{B}$, now the $\beta^{*}$ and $r^{*}$ can be derived asymptotically. It is clear that in the finite system, with the same threshold, the fraction between the number of selected relays and available relays, and equivalently the number of selected relays, varies a lot. Although it may be possible to get the optimal threshold under different metrics, another scheme, "Fraction Selection (FS)", is inspired by the $\beta^{*}$ in the asymptotic result, which always selects a fixed proportion of relays with the largest channel power gains to the destination according to $\bar{B}$.

Fig. 3 presents the outage performance vs. different discrete fractions $(\beta \mathrm{s})$, equivalently different fixed numbers of selected relays $N$, under FS in the DF networks. It is assumed there are

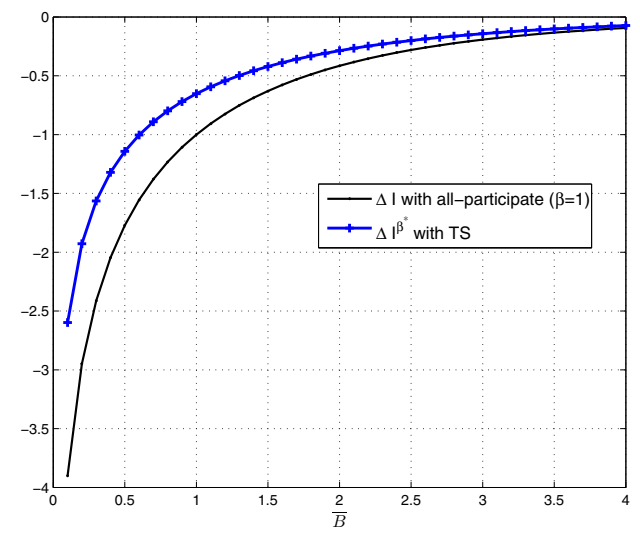

Fig. 2. capacity differences campared with optimal beamforming under two different schemes.

10 relays can decode the message from the source $(M=10)$ and the total transmit energy $E_{r}$ in Phase II is always 1 . Outage capacity is used as the metric and the horizontal axis indicates all available choices of $\beta$ under the particular finite case. From the simulated results, under the same $\bar{B}$, the performance of the corresponding discrete $\beta$ in asymptotic result of Theorem 1 accurately predicts that in the finite case, that is, the $\beta$ achieving the best performance among $\{1 / M, 2 / M, \ldots, 1\}$ according to Theorem 1 also enjoys the best outage performance out of all choices of $\beta$ under FS. For example, when $\bar{B}=1.1, \beta^{*}$ is 0.5 roughly from Fig. 1, and in this simulation with finite relays, the $\beta$ which accomplishes best performance is 0.5 as well. Cases are similar among different $\bar{B}$ s. Furthermore, when $\bar{B}$ is small, the gap between using optimal number of relays and using all of them $(\beta=1)$ is quite large.

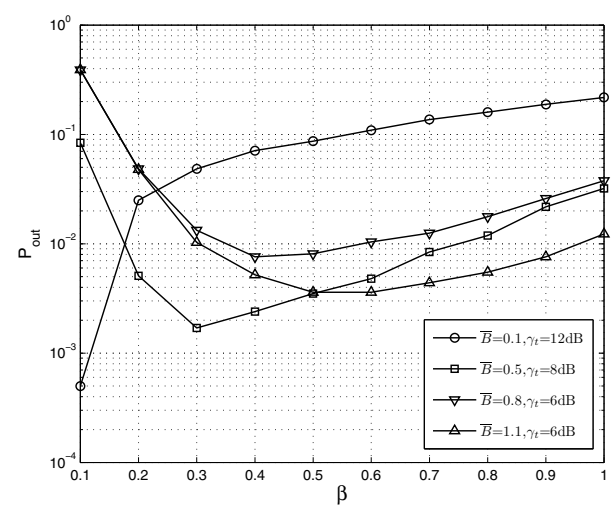

Fig. 3. Outage Probability of DF network for different $\beta$ s with $M=10$, $R=1.7$.

\section{FRACTION SELECTION IN THE AMPLIFY-AND-FORWARD NETWORK}

Theorem 1 is an asymptotic result for the DF networks. We try to explore what other areas can benefit from this result. Different from the relays of DF, all those in AF networks are 
considered to be available in Phase II. Assume the number of available relays in Phase II is always $M$, while all channels, noise contributions, CSI conditions and number of available feedback bits $(B)$ are the same as Section II. Similarly with the SNR expression in (8) of [2], if $N$ out of $M$ relays are selected and denoted as $i_{1}, i_{2}, \ldots, i_{N}\left(1 \leq i_{1}<i_{2} \ldots<i_{N} \leq M\right)$, with $i_{n}$ th relay weighted the $n$th entry of a $N \times 1$ precoding vector $\mathbf{w}_{j}$, then the received SNR for decision becomes

$$
\gamma_{r}=\frac{\left|\sum_{n=1}^{N} \frac{E_{s} E_{r} g_{i_{n}} h_{i_{n}}}{\sqrt{E_{s}\left|g_{i_{n}}\right|^{2}+N_{0}}} w_{j, n}\right|^{2}}{1+\sum_{n=1}^{N}\left|\frac{\sqrt{E_{r}} h_{i_{n}}}{\sqrt{E_{s}\left|g_{i_{n}}\right|^{2}+N_{0}}} w_{j, n}\right|^{2}} \gamma_{t},
$$

where $\gamma_{t}=1 / N_{0}$ and $w_{j, n}$ is the $n$-th entry of $w_{j}$.

All relays seem to be coupled together and it's hard to differentiate individual relay effort. However, equation (11) in [2] has found that this received SNR expression can have the same form as that in the TDMA scheduled AF system when the optimal beamforming vector applies. In other words, if optimal beamforming vector is available, each relay contribution in received SNR is equivalent to "a single relay contribution in the network which transmits the message with full energy $E_{r}$ in the second phase". Motivated by this interesting fact, we order all the relays according to their contribution as the single relay transmitting the message with full energy in Phase II, and then apply FS. The only difference between FS in AF mode and DF mode is that in AF network, the destination which has global CSI order all the relays according to

$$
s n r_{m}=\frac{E_{s} E_{r}\left|g_{m}\right|^{2}\left|h_{m}\right|^{2}}{E_{s}\left|g_{m}\right|^{2}+E_{r}\left|h_{m}\right|^{2}+N_{0}}
$$

decreasingly, i.e., $s n r_{k_{1}}>s n r_{k_{2}}>\ldots>s n r_{k_{M}}$. Pick the largest $N$ relays in the snr ordering (however, ordering among the selected relays is not necessary). The other steps are just the same as that in DF.

In Fig. 4, the outage probability in AF network employing FS is shown. The number of all the available relay is also $M=10$, and we keep $E_{s}=1$ and $E_{r}=1$. Interestingly, $\beta^{*}$ in the AF network with finite relays is consistent with that in Theorem 1 as well. Additionally, allowing using a subset of relays can also achieve large gain compared with all-participate case $(\beta=1)$ when $\bar{B}$ is small and single-relay selection when $\bar{B}$ is no less than 0.5 . For example, in these four simulation curves, the gaps between using optimal $\beta$ s that accomplish best performance and $\beta=1$ are more than $10 \mathrm{~dB}$ under particular $\gamma_{t}$ when $\bar{B}$ are $0.1,0.5,0.8$, respectively. As $\bar{B}$ goes beyond 0.5 , FS can attain gains more than $10 \mathrm{~dB}$ under particular $\gamma_{t}$ as well compared with singly-relay selection.

\section{CONCLUSION}

Employing RVQ for the precoding matrix design, we specify an asymptotically optimal relay selection scheme, Threshold Selection, which chooses relays for transmission according to the power gains of their corresponding channels to the destination and guarantees a fixed fraction of selected relays almost surely, and obtain the asymptotic capacity, the optimal threshold and fraction in the DF network. In the finite system, Fraction Selection is inspired which utilizes the asymptotic

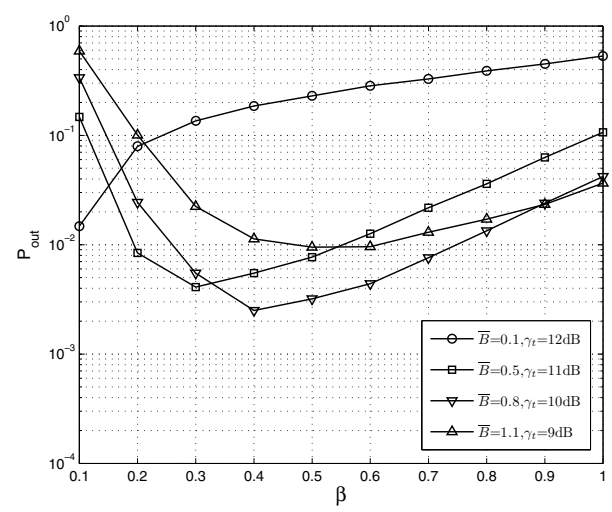

Fig. 4. Outage Probability of AF network for different $\beta$ s with $M=10$, $R=1.5$.

optimal fraction. Given the gap between using optimal fraction of relays and using all relays, it is certainly meaningful to employ just a subset of relays when the resource for feedback is very limited. Simulation results show that it is beneficial to use the optimal $\beta$ to obtain improved performance under FS scheme after power gain ordering and the "snr" ordering in finite-size DF and AF networks, respectively.

\section{REFERENCES}

[1] J. N. Laneman and G. W. Wornell, "Distributed space-time-coded protocols for exploiting cooperative diversity in wireless networks," IEEE Trans. Inform. Theory, vol.49, no. 10, pp. 2415-2425, Nov. 2003.

[2] Y. Zhao, R. Adve, and T. J. Lim, "Beamforming with limited feedback in amplify-and-forward cooperative networks," IEEE Tran. Wireless Commun., vol. 7, no. 12, pp. 5145-5149, Dec. 2008.

[3] Y. Jing and H. Jafarkhani, "Single and multiple relay selection schemes and their achievable diversity orders," IEEE Tran. Wireless Commun., vol. 8, no. 3, pp. 1414-1423, Mar. 2009.

[4] A. Bletsas, H. Shin, and M. Z. Win, "Outage optimality of opportunistic amplify-and-forward relaying," IEEE Commun. Lett., vol. 11, pp. 261263, Mar. 2007.

[5] D. J. Love and R. W. Heath, Jr., "Grassmannian beamforming for multiple-input multiple-output wireless systems," IEEE Trans. Inform. Theory, vol. 49, no. 10, pp. 2735-2745, Oct. 2003.

[6] W. Santipach and M. Honig, "Capacity of a multiple-antenna fading channel with a quantized precoding matrix," IEEE Trans. Inform. Theory, vol. 55, no. 3, pp. 1218-1234, Mar. 2009.

[7] C. K. Au-Yeung and D. J. Love, "On the performance of random vector quantization limited feedback beamforming," IEEE Trans. Wireless Commun., vol. 6, no. 2, pp. 458-462, Feb. 2005.

[8] M. Z. Win and J. H. Winters, "Virtual branch analysis of symbol error probability for hybrid selection/maximal-ratio combining in rayleigh fading," IEEE Trans. Commun., vol. 49, no. 11, pp. 1926-1934, Nov. 2001.

[9] R. Madan, N. B. Mehta, A. F. Molisch, and J. Zhang, "Energy-efficient cooperative relaying over fading channels with simple relay selection," IEEE Trans. Wireless Commun., vol. 7, no. 8, pp. 3013-3025, Aug. 2008.

[10] Y. Jing and H. Jafarkhani, "Network beamforming using relays with perfect channel information," submitted for publication, 2006. [Online] Available: http://arxiv.org/pdf/0804.1117v1.

[11] A. T. James, "Distributions of matrix variates and latent roots derived from normal samples," Annals of Mathematical Statistics, vol. 35, pp. 475-501, June 1964. 